\title{
Alterations in hepatic lobar function in regenerating rat liver
}

\section{András Fülöp, $\mathrm{MD},{ }^{a}$ András Budai, $\mathrm{MD},{ }^{a}$ Zoltán Czigány, $\mathrm{MD},{ }^{a}$ Gábor Lotz, MD, PhD, ${ }^{b}$ Katalin Dezső, MD, PhD, ${ }^{c}$ Sándor Paku, PhD, DSc, ${ }^{c, d}$ László Harsányi, $\mathrm{MD}, \mathrm{PhD},{ }^{a}$ and Attila Szijártó, $\mathrm{MD}, \mathrm{PhD}^{a, *}$}

a 1st Department of Surgery, Semmelweis University, Budapest, Hungary

${ }^{\mathrm{b}}$ 2nd Department of Pathology, Semmelweis University, Budapest, Hungary

c1st Department of Pathology and Experimental Cancer Research, Semmelweis University, Budapest, Hungary

${ }^{\mathrm{d}}$ Tumor Progression Research Group, Joint Research Organization of the Hungarian Academy of Sciences and Semmelweis University, Budapest, Hungary

\section{A R T I C L E I N F O}

Article history:

Received 29 January 2015

Received in revised form

16 March 2015

Accepted 9 April 2015

Available online 15 April 2015

Keywords:

Bile flow

Indocyanine green

Liver function

Liver regeneration

Portal vein ligation

\section{A B S T R A C T}

Background: Ligation of a branch of the portal vein redirects portal blood to nonligated lobes resulting in lobar hypertrophy. Although the effect of portal vein ligation on liver volume is well documented, the parallel alterations in liver function are still the subject of controversy. Our aim was to assess the time-dependent reactions of regional hepatic function to portal vein ligation by selective biliary drainage.

Methods: Male Wistar rats $(n=44)$ underwent $80 \%$ portal vein ligation. Before the operation as well as 1, 2, 3, 5, and $7 \mathrm{~d}$ after circulation, morphology and function (laboratory blood test; hepatic bile flow; plasma disappearance rate of indocyanine green; and biliary indocyanine green excretion) of the liver were examined.

Results: Although portal vein ligation affected liver circulation and morphology to a great extent, serum albumin levels, bilirubin levels, and total hepatic bile flow did not change significantly after the operation. Nevertheless, plasma disappearance rate and biliary indocyanine green excretion indicated a temporary impairment of total liver function with the lowest value on the second day and normalization by the fifth day. Bile production and biliary indocyanine green excretion of ligated lobes decreased rapidly after the operation and remained persistently suppressed, whereas the secretory function of nonligated lobes-after a temporary decline-showed a greater increase than the weight of the lobes. Conclusions: Portal vein ligation induced temporary impairment of total liver function, followed by rapid recovery mainly by reason of increase in the function of nonligated lobes. Functional increase in nonligated lobes was more pronounced than suggested by the degree of volume gain.

(c) 2015 Elsevier Inc. All rights reserved.
1.

\section{Introduction}

Curative liver resection provides the best survival rate for patients with liver malignancies [1]. Extended hepatectomy is usually required to achieve negative margins. Excessive removal of the hepatic parenchyma, however, often leads to postoperative liver failure. The most widely used method to overcome this problem is preoperative enlargement of the

\footnotetext{
* Corresponding author. 1st Department of Surgery, Semmelweis University, Üllöi út 78, H-1082 Budapest, Hungary. Tel.: +36 208258925 ; fax: +36208252162 .

E-mail address: szijartoattila@gmail.com (A. Szijártó). 0022-4804/\$ - see front matter @ 2015 Elsevier Inc. All rights reserved.
} 
future liver remnant (FLR) volume by portal vein occlusion (PVO) techniques, such as portal vein embolization, two-stage hepatectomy combined with portal vein ligation, and more recently associating liver partition with portal vein ligation for staged hepatectomy. These surgical procedures are applied to redirect portal blood flow away from the liver lobes designated for resection, toward the anticipated FLR resulting in FLR hypertrophy (regeneration) [2].

Currently, computed tomography (CT) volumetry is the standard method for determining whether sufficient regeneration is present after PVO. Nevertheless, increase in the FLR volume (morphologic regeneration) does not necessarily reflect the actual alterations of the FLR function (functional regeneration) [3]. Although the morphologic phenomena have been widely discussed, little is known about the functional alterations occurring after PVO because of the lack of an ideal quantitative test, which would represent multiple aspects of the liver function and would be able to assess FLR function selectively. There are two contradictory theories in the literature. Some studies postulate that liver regeneration is promoted at the expense of liver function, resulting in prolonged and less functional regeneration compared with the rapid increase in FLR volume [4-6]. Other works, based on nuclear imaging techniques (i.e., hepatobiliary scintigraphy [7] or hepatocyte mass scintigraphy $[8,9])$, indicate that increase in FLR function is more pronounced than implied by the degree of morphologic regeneration. This hypothesis, however, has not yet been confirmed by other widely accepted quantitative liver function tests.

Over the last decades, several quantitative liver function tests have been developed, of which the indocyanine green (ICG) clearance test is the most common. ICG is a fluorescent tricarbocyanine dye exclusively eliminated by the liver without metabolism and enterohepatic recirculation [10]. Although ICG is not metabolized, it follows a path of intracellular transport similar to several exogenous and endogenous molecules; its disappearance from the blood, therefore, provides indirect information about the overall function of the liver. The main limitation of the test is that it does not take into account regional variations in liver quality that may occur after PVO.

Selective biliary drainage, however, enables us to assess biliary ICG excretion selectively in FLR. Literary data have demonstrated that biliary ICG excretion is an excellent indicator of liver function or dysfunction in various pathologic conditions such as liver ischemia-reperfusion, liver transplantation, or severe septic state [11-13]. Furthermore, the capacity of the liver to excrete ICG accurately reflects the intracellular adenosine triphosphate (ATP) level and hence the energy status of hepatocytes, which are among the most decisive factors in terms of functionality and organ viability [14].

The aim of the present study was to selectively assess the time-dependent reactions of regional hepatic function to portal vein ligation by selective biliary drainage and assessment of biliary ICG excretion compared with the conventional parameters of liver regeneration and hepatic circulation.

\section{Materials and methods}

The experimental design was regulated in accordance with the National Institutes of Health guidelines for animal care and was approved by the Committee on Animal Experimentation of Semmelweis University (license number: PEI/001/313-4/2014). Male Wistar rats weighing 200-250 g were used (Semmelweis University, Central Animal Facility, Budapest, Hungary). Standard rat chow and water were provided ad libitum. Before the experiment, the rats were fasted overnight to minimize the effect of food ingestion on the liver blood and bile flow.

\subsection{Experimental design and operative procedure}

Portal vein ligation was performed as previously described [15]. Briefly, under general anesthesia, induced by intraperitoneal injection of ketamine $(75 \mathrm{mg} / \mathrm{kg})$ and xylazine $(7.5 \mathrm{mg} / \mathrm{kg})$, the portal branches supplying the median, left lateral, and caudate lobes-approximately $80 \%$ of the total liver mass-were ligated. After operation, the animals were returned to their cages. Rats $(n=36)$ were randomly allocated into groups based on the length of the recovery period. Six animal groups were examined as follows: on the preoperative day (day 0 , control group) and on postoperative days 1, 2, 3, 5, and 7 ( $n=6$ per time point), respectively. Additional eight animals were used to determine the size of liver lobules before $(n=4)$ and $7 \mathrm{~d}$ after $(n=4)$ PVO (Fig. 1).

At the indicated time points, animals were reanesthetized and a 22-gauge polyethylene catheter was placed into the right jugular vein for maintenance of anesthesia $(25-\mathrm{mg} / \mathrm{kg} / \mathrm{h}$ ketamine and $2.5-\mathrm{mg} / \mathrm{kg} / \mathrm{h}$ xylazine) and for the administration of saline infusion $(4 \mathrm{~mL} / \mathrm{kg} / \mathrm{h})$ as well as ICG. Another 22gauge polyethylene catheter was inserted into the left carotid artery for hemodynamic measurements. After relaparotomy, surface probes of the laser Doppler flowmeter were placed on the liver's superior right lateral (SRL) and left lateral lobes (LLL). After registration of the liver microcirculatory flow, the bile ducts of portal vein ligated (PVL) and portal vein nonligated (PVNL) lobes were selectively cannulated to assess the bile flow. ICG in a dose of $1.5 \mathrm{mg} / \mathrm{mL}$ (ICG-PULSION; PULSION Medical Systems, Munich, Germany) was injected, and then plasma disappearance rate (PDR) and biliary excretion of the dye were determined. After a 150-min period of bile collection, the portal vein was directly punctured with a 24-gauge needle to measure the portal pressure. At the end of the measurements, rats were exsanguinated via the right ventricular puncture and liver samples were excised (Fig. 1).

\subsection{Assessment of hemodynamics and liver microcirculation}

Blood pressure was measured by an invasive blood pressure monitoring system (Kent Scientific Corporation, Torrington, CT) via the cannulated right carotid artery. Portal pressure was evaluated by direct puncture of the portal vein (Kent Scientific Corporation). Liver microcirculation was assessed before ICG administration using a laser Doppler monitor (DRT4 device with DP1T surface probe; Moor Instruments Ltd, London, United Kingdom). Surface probes were placed on fixed locations of the liver's SRL (PVNL) and LLL (PVL). At least four measurements were performed at different sites of the lobes ( $5 \mathrm{~min}$ for each), and the mean of the measurements was calculated. 


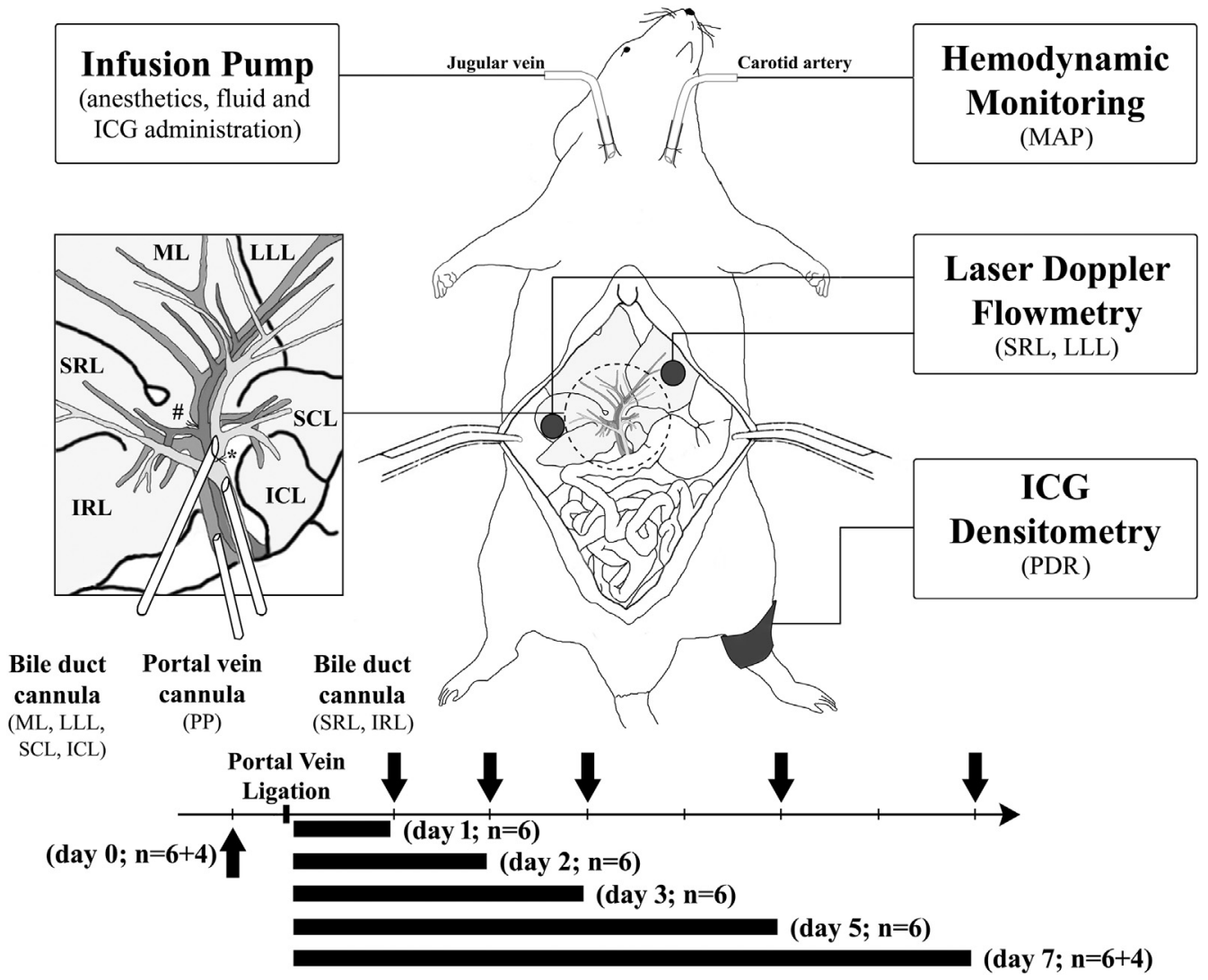

Fig. 1 - Experimental design. At the indicated time points (arrows), six groups of animals were used to determine the alterations in circulation, morphology, and functional capacity of the liver lobes after portal vein ligation. MAP, mean arterial pressure; PP, portal pressure; IRL, inferior right lobe; ML, median lobe; SCL, superior caudate lobe; ICL, inferior caudate lobe. \# indicates portal vein ligation; * indicates selective bile duct cannulation.

\subsection{Assessment of morphologic alterations}

\subsubsection{Liver weight measurement}

Wet weight of liver lobes was measured separately using laboratory scale (Mettler-Toledo AG 245; Mettler-Toledo, LLC, Columbus, $\mathrm{OH}$ ). The relative weight of lobes to body weight (BW) was then calculated and expressed as liver weight (gram) per 100-g BW.

\subsubsection{Histologic and immunohistochemical analysis}

Histologic samples were harvested from SRL (PVNL) and LLL (PVL) from identical sites. The excised samples were fixed in $4 \%$ neutral-buffered formalin and embedded in paraffin. Sections of 3-5 $\mu \mathrm{m}$ thickness were examined under light microscope after hematoxylin and eosin staining. Necrosis was assessed by morphologic features and graded on a scale of 0-4 as described by Suzuki et al. [16]: grade 0-absence of necrosis; grade 1-single-cell necrosis; grade $2-30 \%$ lobular necrosis; grade 3-60\% lobular necrosis; and grade 4-more than $60 \%$ lobular necrosis. Apoptotic cell death and mitotic activity were determined by immunohistochemical staining procedures. Primary antibodies against active caspase-3 (Cell Signaling Technology, Beverly, MA) and Ki-67 (DAKO, Glostrup, Denmark) were used, respectively. Avidin-biotin-peroxidase complex immunohistochemical method was performed for antigen retrieval. Labeling index was defined as the mean number of positive cells per 10 visual fields $(\times 200)$.

\subsubsection{Liver lobule size determination}

In a separate study, the size of liver lobules was determined before $(n=4)$ and $7 \mathrm{~d}$ after portal vein ligation $(n=4)$ as previously described $[17,18]$. Briefly, hepatic veins and sinusoids were filled up through the inferior vena cava by a fluorescent dye containing polystyrene resin. The liver surface was monitored by stereomicroscope, and the filling was stopped when the resin filled up the sinusoids partially. SRL (PVNL) and LLL (PVL) were removed and examined with inverted microscope (Nikon TE200; Nikon, Tokyo, Japan). Surface images of the lobes were captured by a Bio-Rad (MRC1024; Bio-Rad, Richmond, CA) confocal system. On the surface pictures, the interlobular borders were outlined by the resin in the sinusoids. Circumference and surface area of the lobules were determined with ImageJ program (NIH, Bethesda, MD).

\subsection{Assessment of functional alterations}

\subsubsection{Conventional laboratory blood tests}

Serum levels of alanine aminotransferase, aspartate aminotransferase, albumin, and total bilirubin (tBil) were determined with an automated clinical chemistry analyzer (Beckman Coulter AU480/2011; Beckman Coulter Inc, Brea, CA). 

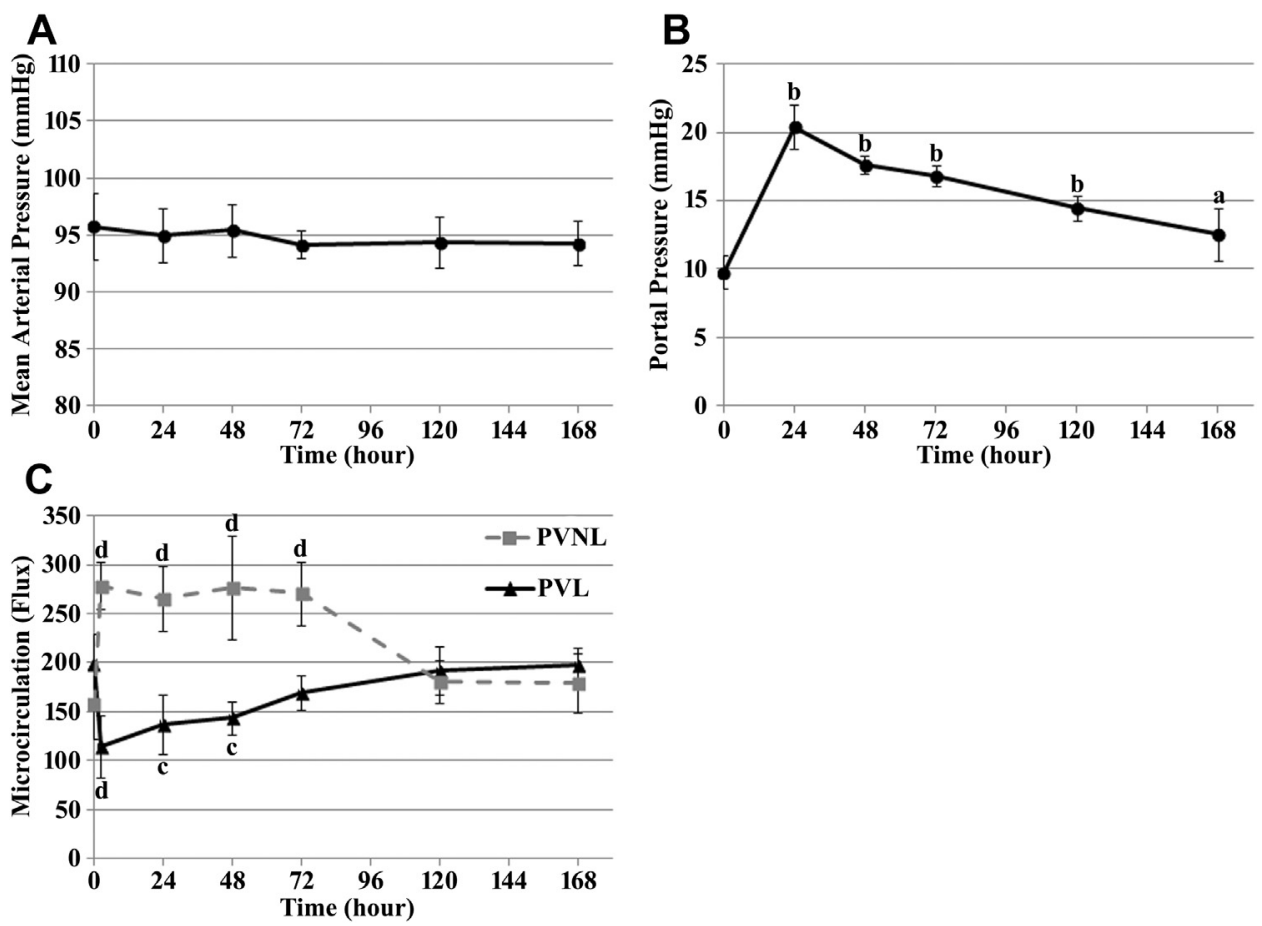

Fig. 2 - Hemodynamics and liver microcirculation. (A) The mean arterial pressure did not significantly change throughout the entire experiment. (B) The portal pressure significantly increased after portal vein ligation and remained elevated until the seventh day. (C) The microcirculatory flow of PVNL lobes immediately increased after the operation and remained elevated until the third day, whereas the microcirculation of PVL lobes was temporarily impaired and became normalized on the third day. Results are given as mean \pm standard deviation, $n=6$ per group: ${ }^{\text {ap }}<0.05$ versus the preoperative level (day 0); ${ }^{b} P<0.001$ versus the preoperative level (day 0 ); ${ }^{c} P<0.05$ versus the preoperative value (day 0 ) of the corresponding lobes; and ${ }^{d} P<0.001$ versus the preoperative value (day 0 ) of the corresponding lobes.

\subsubsection{Plasma disappearance rate}

PDR was determined by means of ICG densitometry using a commercial analyzer (PC5000 LiMON; PULSION Medical Systems) and a corresponding neonatal finger probe (PV50200 disposable sensor for neonates; PULSION Medical Systems), which was attached to the left hind limb with a bandage. ICG $(1.5 \mathrm{mg} / \mathrm{mL})$ was injected at the dose of $1 \mathrm{~mL} / \mathrm{kg}$ via the right jugular vein. After a registration period of $6 \mathrm{~min}$, the device calculated PDR.

\subsubsection{Bile production and biliary ICG excretion}

To determine bile production and biliary ICG excretion of the liver, selective biliary drainage was performed. Bile was collected from the PVL and PVNL lobes separately, first at 5-min intervals (from 0-40 $\mathrm{min}$ ), then at 10-min intervals (from 40-150 $\mathrm{min}$ ) into preweighed, light-protected test tubes. Bile volume from each collection was measured gravimetrically, assuming a specific gravity of 1.0 [19]. Bile production was normalized to both BW $(\mu \mathrm{L} / \mathrm{min} / \mathrm{kg}$ BW) and liver weight ( $\mu \mathrm{L} / \mathrm{min} / \mathrm{g}$ liver). After measurement of the bile volume, the samples were centrifuged. The supernatant was diluted with distilled water (1:4) and analyzed for ICG concentration using Zenith 200rt spectrophotometer (Anthos Labtec Instruments $\mathrm{GmbH}$, Salzburg, Austria) at $805 \mathrm{~nm}$ [13]. Maximal biliary ICG concentration $\left(\mathrm{C}_{\max }\right)$ and the corresponding time $\left(\mathrm{T}_{\max }\right)$ were determined from the concentration curves. Furthermore, biliary ICG excretion was calculated by multiplying the biliary ICG concentrations by the collected bile volume. Then the biliary ( ICG $_{\mathrm{EX}}$ ) was expressed as a percentage of excreted ICG at the first $20 \mathrm{~min}$ from the injected dose.

\subsection{Statistical analysis}

Values were expressed as means \pm standard deviation. Normality and homoscedasticity of the data were tested. Statistical analysis was performed with the analysis of variance test and the Bonferroni correction post hoc test. For analysis of nonparametric data, the Kruskal-Wallis $\mathrm{H}$ test was applied. Differences were considered significant when $P<0.05$. Calculations were performed with IBM SPSS Statistics 20.0 software (IBM Corporation, Armonk, NY).

\section{Results}

\subsection{Hemodynamics and liver microcirculation}

Mean arterial pressure remained around the baseline level during the experiments (Fig. 2A). Portal pressure increased significantly $1 \mathrm{~d}$ after the operation $(P<0.001$; day 1 versus day $0)$ and remained elevated until the seventh day $(P=0.02$; day 7 versus day 0; Fig. 2B). 

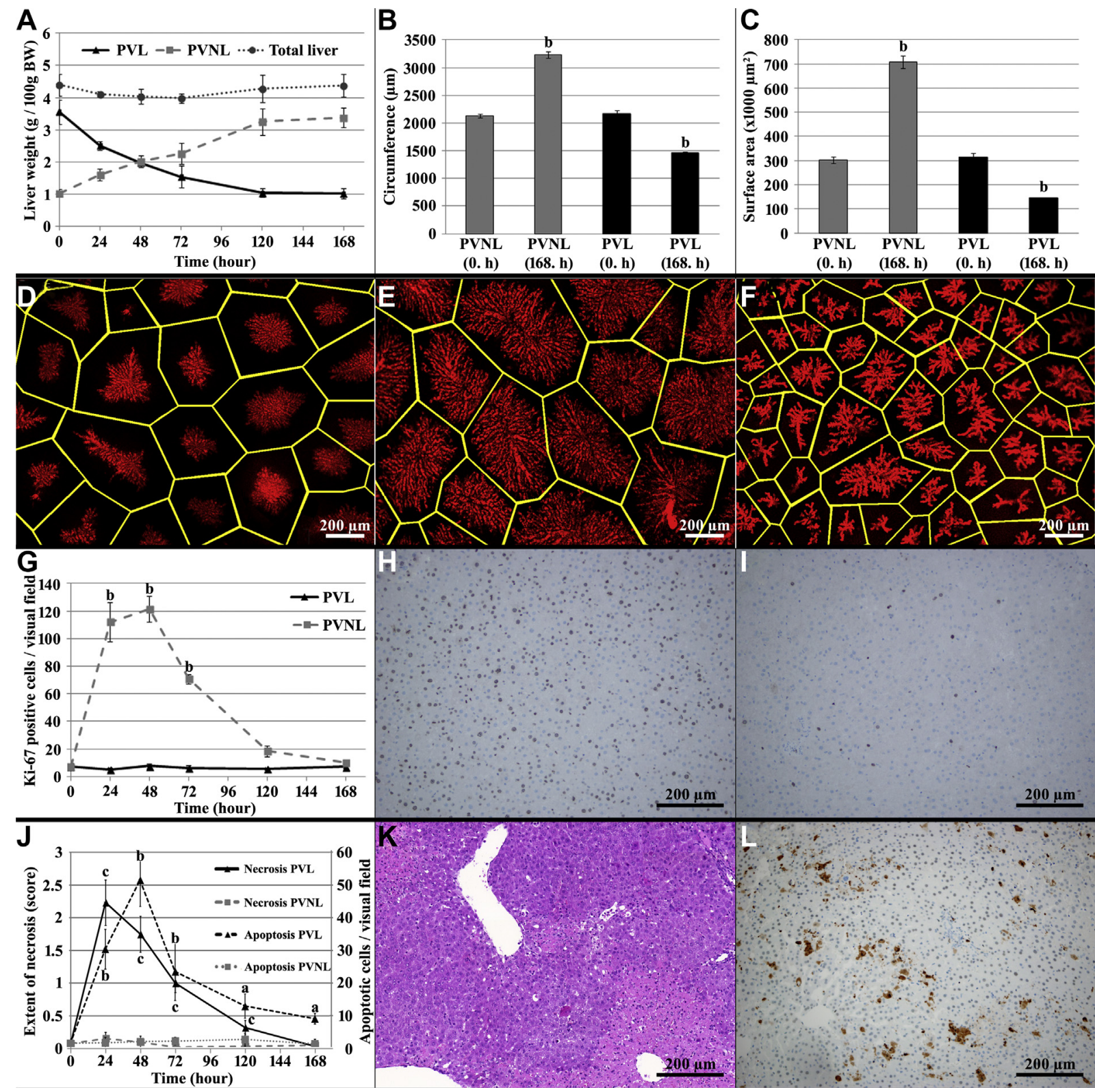

Fig. 3 - Morphologic alterations. (A) Changes in weight of total liver, PVL, and PVNL liver lobes at the examined time points. The weight of PVNL lobes increased considerably after portal vein ligation in association with a significant enlargement in average circumference (B and $E$ ) and surface area ( $C$ and $E$ ) of the liver lobules compared with the preoperative values (B-D). In contrast, PVL lobes shrunk considerably, characterized by decreased lobular circumference (B and F) and surface area (C and F). Yellow lines indicate the interlobular borders in parts D-F. Histomorphologic characteristics of the PVL and PVNL lobes after the operation. Mitotic activity of hepatocytes significantly increased in PVNL lobes, with peak response on the second day (G). In the PVNL lobes, Ki-67 immunohistochemistry (brown stain) showed a high expression level of Ki-67 in cell nuclei (H), whereas the number of Ki-67 positive cells in PVL lobes did not increase (I). In PVL lobes, the extent of necrotic areas and the number of apoptotic cells significantly increased after the operation with peak response on the first and second day, respectively (J). Wide necrotic areas were seen around the central vein $1 \mathrm{~d}$ after the operation (K). At this time, apoptosis was also frequently present (caspase- 3 immunohistochemistry, L). Results are given as mean \pm standard deviation, $n=6$ per group: ${ }^{a} P<0.05$ versus the preoperative value (day 0 ) of the corresponding lobes; ${ }^{b} P<0.001$ versus the preoperative value (day 0 ) of the corresponding lobes; and ${ }^{C} P<0.05$ versus the preoperative value (day 0 ) of the corresponding lobes. (Color version of figure is available online.)

Microcirculatory blood flow of the PVNL lobes increased immediately after portal vein ligation and remained elevated until the third day $(P<0.001$; day 3 versus day 0$)$. On the fifth day, it returned to the values detected before the operation. In contrast, in PVL lobes, the microcirculatory flow decreased by almost $50 \%$ after the operation. Then a gradual recovery was 
Table - Laboratory blood test and ICG clearance test.

\begin{tabular}{|c|c|c|c|c|c|c|}
\hline Measured parameters & Day 0 & Day 1 & Day 2 & Day 3 & Day 5 & Day 7 \\
\hline Aspartate aminotransferase (U/L) & $109.33 \pm 17.39$ & $936.5 \pm 270.77^{*}$ & $561.17 \pm 140.69$ & $179.67 \pm 73.08$ & $104.17 \pm 8.5$ & $103.67 \pm 13.92$ \\
\hline Alanine aminotransferase (U/L) & $55.67 \pm 12.68$ & $592.5 \pm 203.63$ & $386.33 \pm 93.8^{*}$ & $86.83 \pm 41.35$ & $58.33 \pm 12.13$ & $56.5 \pm 10.65$ \\
\hline $\mathrm{tBil}(\mu \mathrm{mol} / \mathrm{L})$ & $1.78 \pm 0.26$ & $1.9 \pm 0.26$ & $1.98 \pm 0.19$ & $1.93 \pm 0.29$ & $1.82 \pm 0.23$ & $1.8 \pm 0.25$ \\
\hline Albumin (g/dL) & $2.23 \pm 0.18$ & $2.15 \pm 0.27$ & $2.5 \pm 0.26$ & $2.33 \pm 0.15$ & $2.41 \pm 0.19$ & $2.21 \pm 0.34$ \\
\hline PDR-ICG $(\% / \mathrm{min})$ & $50.97 \pm 6.56$ & $27.9 \pm 4.69^{*}$ & $23.55 \pm 5.19^{*}$ & $35.3 \pm 7.29^{*}$ & $46.83 \pm 5.17$ & $51.17 \pm 7.45$ \\
\hline
\end{tabular}

observed, and on the third day, the microcirculatory flow of the lobes did not differ significantly from the preoperative value $(P=0.89$; day 3 versus day 0 ; Fig. $2 C)$.

\subsection{Morphologic alterations}

\subsubsection{Liver weight}

Although the total liver weight remained unchanged, the weight of PVNL lobes significantly increased after the operation and reached a plateau between the fifth and seventh days at approximately $329 \%$ of the preoperative value. On the other hand, PVL lobes considerably shrunk and reached about $29 \%$ of their original weight by the end of the experiment (Fig. 3A).

\subsubsection{Liver histology}

Portal vein ligation led to increased mitotic activity in PVNL lobes with peak response on the second day $(P<0.001$; day 2 versus day 0 ). Then the number of proliferating hepatocytes gradually decreased, and on the fifth day, the incidence of mitosis did not significantly differ from that seen before the operation ( $P=0.2$; day 5 versus day 0; Fig. 3G-I.

In PVL lobes, extensive necrotic areas occurred around the central veins $1 \mathrm{~d}$ after the operation. A high number of leukocytes were observed within the necrotic region. In addition to necrosis, the number of apoptotic cells also increased, with the peak appearing on the second day $(P<0.001$; day 2 versus day 0$)$. On the seventh day, normal hepatic histology was observable with no signs of necrosis, whereas the frequency of apoptosis remained elevated $(P=0.03$; day 7 versus day 0$)$. At this time, the portal triads seemed to be contracted with thickened portal veins, collapsed bile ducts, and enlarged hepatic arteries (Fig. 3J-L).

\subsubsection{Liver lobule size}

Weight gain of the PVNL lobes was associated with 1.52- and 2.35-fold increase in the average circumference and surface area of the liver lobules, respectively. By contrast, the lobules of PVL lobes shrunk considerably, and the lobular circumference and surface area decreased by 0.32 - and 0.54 -fold, respectively (Fig. 3B-F).

\subsection{Functional alterations}

\subsubsection{Laboratory blood tests}

No significant alterations were observable regarding the serum tBil and albumin levels. Portal vein ligation, however, resulted in a transient elevation of transaminases that peaked after the first day (aspartate aminotransferase: $P=0.01$; day 1 versus day 0; alanine aminotransferase: $P<0.001$; day 1 versus day 0 ) and normalized on the third day (Table).

\subsubsection{Plasma disappearance rate}

Portal vein ligation resulted in a significant reduction in PDR. The liver's clearance function was minimal around the second day (PDR: $P<0.001$; day 2 versus day 0 ), but a gradual recovery was observable thereafter, and on the fifth day, the PDR did not show significant difference from the preoperative level (PDR: $P=0.93$; day 5 versus day 0; Table).

\subsubsection{Bile production}

Bile production normalized to BW showed a sharp increase in PVNL lobes $1 \mathrm{~d}$ after the operation. Thereafter, further gradual increment was observable, as a result of which bile production of the lobes reached about $380 \%$ of the preoperative value on the seventh day. By contrast, in PVL lobes, the bile production per BW kilogram gradually decreased until the seventh day. Owing to a balance between the bile production of PVNL and PVL lobes, the total liver bile flow remained unchanged throughout the entire experiment (Fig. 4A).

When bile production was normalized to liver weight, the bile flow of PVNL lobes significantly increased, with peak response visible on the second day (1.48-fold increase). On the contrary, bile production of the PVL lobes showed gradual decrease until the third day (0.7-fold decrease). Between the fifth and seventh days, the bile production per gram liver returned almost to the preoperative level both in PVNL and PVL lobes (PVNL: $P=0.91$; day 7 versus day 0; PVL: $P=0.87$; day 7 versus day 0$)$. However, at these time points, the bile production per gram liver of PVNL lobes remained significantly higher in comparison with that of PVL lobes (day 5: $P<0.001$; PVNL versus PVL; day 7: $P=0.01$; PVNL versus PVL; Fig. 4B).

\subsubsection{Biliary ICG excretion}

$\mathrm{C}_{\max }$ and $\mathrm{T}_{\max }$ were determined via analysis of the biliary ICG concentration curves. In PVNL lobes, $\mathrm{T}_{\max }$ was significantly prolonged $(P=0.003$; day 2 versus day 0$)$ and $C_{\max }$ was significantly reduced ( $P=0.049$; day 2 versus day 0$) 2 \mathrm{~d}$ after the operation. Thereafter, a gradual recovery was observed, and on the seventh day, $\mathrm{T}_{\max }$ returned to the preoperative level $(P=0.34$; day 7 versus day 0$)$, whereas $C_{\max }$ exceeded the value observed before operation, although the difference was not significant ( $P=0.27$; day 7 versus day 0$)$. On the first $2 d$, the biliary ICG concentration curves of PVL lobes changed similarly to the curves seen in case of the PVNL lobes. However, 

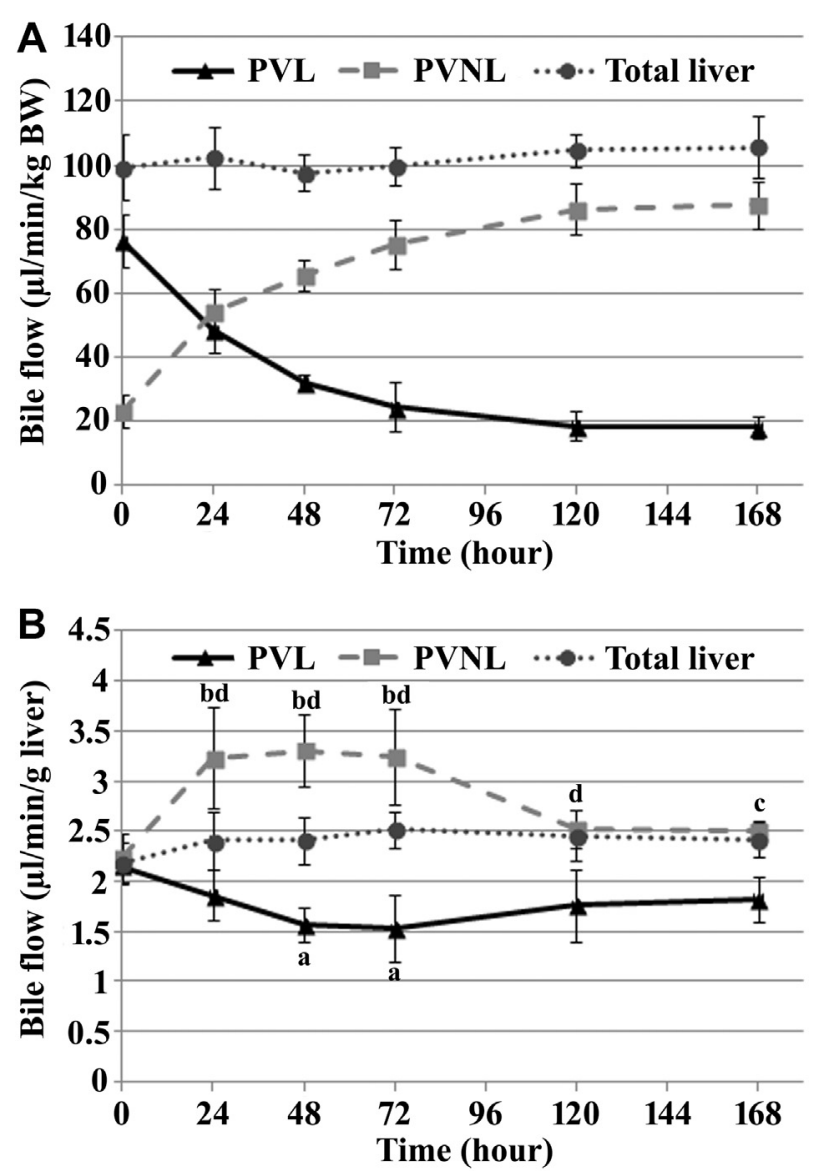

Fig. 4 - Bile production. (A) The bile flow of total liver normalized to BW did not change after portal vein ligation. In contrast, the bile production of PVNL lobes sharply increased, whereas the bile flow of PVL lobes substantially decreased after the operation. (B) The bile production of PVNL lobes normalized to the liver weight significantly increased, whereas that of PVL lobes gradually decreased until the third day. Between the fifth and seventh days, the bile production per gram liver almost returned to the preoperative value of both the PVL and PVNL lobes. However, at these time points, the bile flow of PVNL lobes remained significantly higher compared with that of PVL lobes. Results are given as mean \pm standard deviation, $n=6$ per group: ${ }^{\text {a }}<0.05$ versus the preoperative value (day 0 ) of the corresponding lobes; ${ }^{\mathrm{b}} \mathrm{P}<0.001$ versus the preoperative value (day 0 ) of the corresponding lobes; ${ }^{\mathrm{C} P}<0.01$ versus $\mathrm{PVL}$ lobes; and ${ }^{d_{P}}<0.001$ versus $P V L$ lobes.

after the second day, $\mathrm{T}_{\max }$ and $\mathrm{C}_{\max }$ did not become normalized and remained significantly inferior as compared with the preoperative values ( $T_{\max }: P=0.01$; day 7 versus day $0 ; C_{\max }$ : $P<0.001$; day 7 versus day 0; Fig. 5A-D).

The biliary ICG $_{\mathrm{EX}}$ of the total liver became temporarily impaired after the operation, showing the lowest value on the second day $(P<0.001$; day 2 versus day 0$)$. Thereafter, the excretory function of the liver gradually recovered, reaching preoperative levels on the fifth day $(P=0.89$; day 5 versus day $0)$. The ICG $\mathrm{EX}_{\mathrm{X}}$ of the PVNL lobes did not change significantly during the first $2 \mathrm{~d}(P=0.33$; day 1 versus day $0 ; P=0.07$; day 2 versus day 0 ). After the second day, the ICG $_{\mathrm{EX}}$ of the lobes sharply increased and reached approximately $394 \%$ of the preoperative level on the seventh day. In contrast, the ICG of PVL lobes gradually decreased and reached about $10 \%$ of its preoperative value at the end of the experiment (Fig. 5E).

\subsection{Comparison between morphologic and functional regeneration of PVNL lobes}

When the alterations in liver weight and liver function (bile production and ICG excretion) were compared, there were noticeable discrepancies between the morphologic and functional regeneration of PVNL lobes. The increment (\% from the baseline) in bile production was more pronounced than the alteration in liver weight at any examined time point. By contrast, the increase in ICG excretion proved to be smaller than the weight gain during the first $2 \mathrm{~d}$ (the difference was not significant). From the third day, however, ICG excretion increased more dramatically than liver weight (Fig. 6).

\section{Discussion}

In the present study, the effects of portal vein ligation on the functional capacity of the hepatic lobes were studied in addition to the liver circulation and conventional parameters of liver regeneration (morphologic regeneration).

In our $80 \%$ portal vein ligation model, although the systemic circulation was not affected, considerable alterations were seen in liver hemodynamics. Immediately after the operation, the portal pressure and microcirculatory blood flow of PVNL lobes showed substantial increase, whereas noteworthy microcirculatory impairment was observed in the PVL lobes. These hemodynamic alterations are considered as an important driving force for the induced morphologic changes [20]. Total cessation of the portal venous inflow led to atrophy of the PVL lobes. This mass loss was fully compensated by the rapid weight gain observable in case of the PVNL lobes; consequently, the total liver weight remained constant. The weight gain in PVNL lobes reached a plateau phase between the fifth and seventh days at approximately 329\% of the baseline, which finding was consistent with previously published data $[5,21,22]$. However, the specific alterations in hepatic lobar structure have not been assessed so far. In the present study, we found that at the end of the regenerative process, the PVNL lobes were characterized by enlarged hepatic lobules. A very similar degree of lobular enlargement was observed by Papp et al. [17], who demonstrated that hypertrophy of the preexisting liver lobules is exclusively responsible for the regenerative liver growth after partial hepatectomy, resulting in a marked change in the liver structure (elongated, more branched portal and central veins, as well as the arborescent shape of zonally expressed enzymes). Based on our observations, similar structural alterations are suspected in PVNL lobes after portal vein ligation, which may considerably affect the functionality of the liver. 


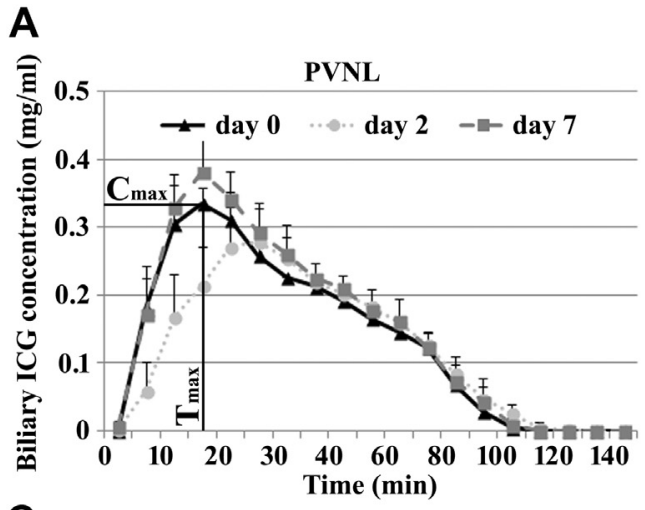

C

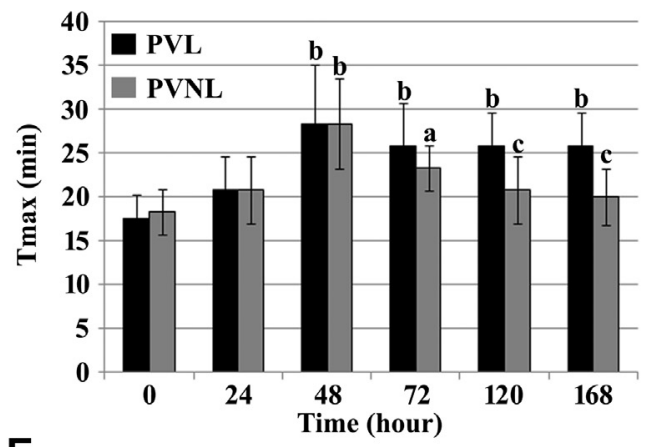

E

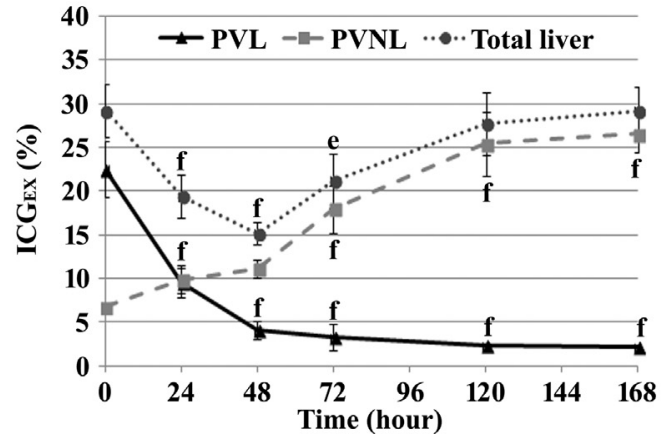

B

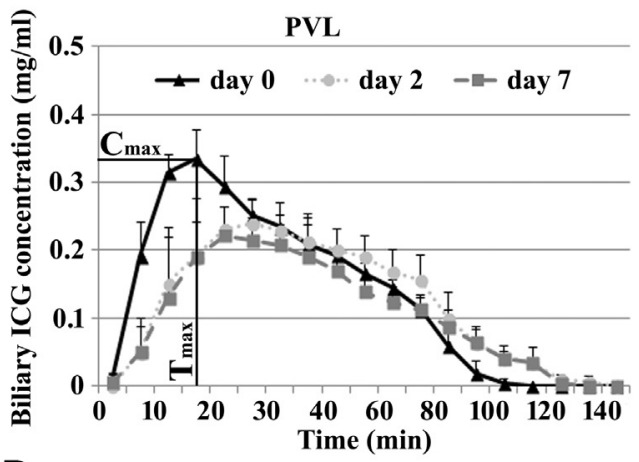

D

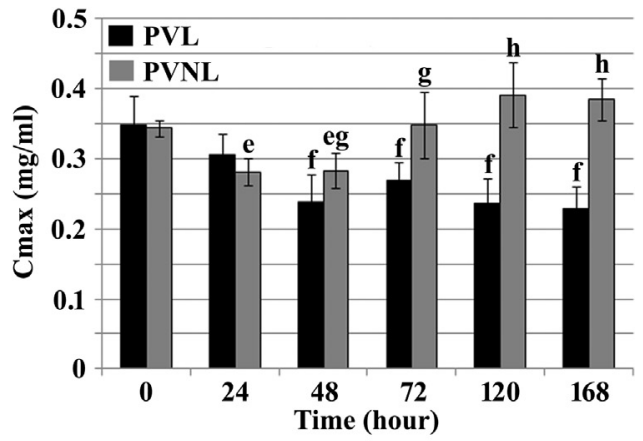

Fig. 5 - Biliary ICG excretion. (A and B) Biliary ICG concentration curves of PVNL and PVL lobes. For the characterization of the individual concentration curves, a new method was introduced, which allowed comparison of the graphs and was suitable for statistical analysis. This involved the $C_{\max }$ and the $T_{\max }(C$ and $D)$. In PVNL lobes, the biliary ICG excretion was significantly prolonged $2 \mathrm{~d}$ after the operation characterized by increased $T_{\max }$ and reduced $C_{\max }$. Thereafter, a gradual recovery could be seen, and on the seventh day, the $T_{\max }$ and $\mathrm{C}_{\max }$ returned to the preoperative level. The biliary ICG excretion of PVL lobes changed similar to that seen in PVNL lobes; however, after the second day, $T_{\max }$ and $\mathrm{C}_{\max }$ did not normalize and remained permanently inferior compared with the preoperative value (day 0 ). (E) Portal vein ligation resulted in a transient impairment of the biliary $\mathrm{ICG}_{\mathrm{EX}}$ of the total liver $2 \mathrm{~d}$ after the operation, with normalization on the fifth day. Although the $\mathrm{ICG}_{\mathrm{EX}}$ of the PVNL lobes did not change significantly during the first $\mathbf{2} \mathrm{d}$, a sharp increase was noticeable thereafter. In contrast, the ICG $\mathrm{Ex}$ of PVL lobes gradually decreased until the end of the experiment. Results are given as mean \pm standard deviation, $n=6$ per group. ${ }^{a} P<0.05$ versus the preoperative value (day 0 ) of the corresponding lobes; ${ }^{b_{P}}<0.01$ versus the preoperative value (day 0 ) of the corresponding lobes; ${ }^{{ }^{C}} P<0.05$ versus PVL lobes; ${ }^{{ } P}<0.05$ versus the preoperative value (day 0 ) of the corresponding lobes; ${ }^{f} P<0.001$ versus the preoperative value (day 0 ) of the corresponding lobes; ${ }^{{ }^{P}}<0.05$ versus PVL lobes; and ${ }^{h_{P}}<0.001$ versus PVL lobes.

To assess the functional consequences of previously mentioned circulatory and morphologic alterations, conventional laboratory blood tests as well as ICG clearance test were performed. According to the routine biochemical parameters (tBil and albumin), liver function remained stable after the operation with only temporary increment in serum transaminase levels, which in fact represents liver injury-necrosis of PVL lobes-rather than real functional deterioration. Nevertheless, the ICG clearance test (PDR) indicated substantial impairment in total liver function $2 \mathrm{~d}$ after the operation, followed by a rapid recovery period. Considering that the ICG clearance test only represents the functional capacity of the total liver without providing information about regional variations, in the present study, we performed selective biliary 


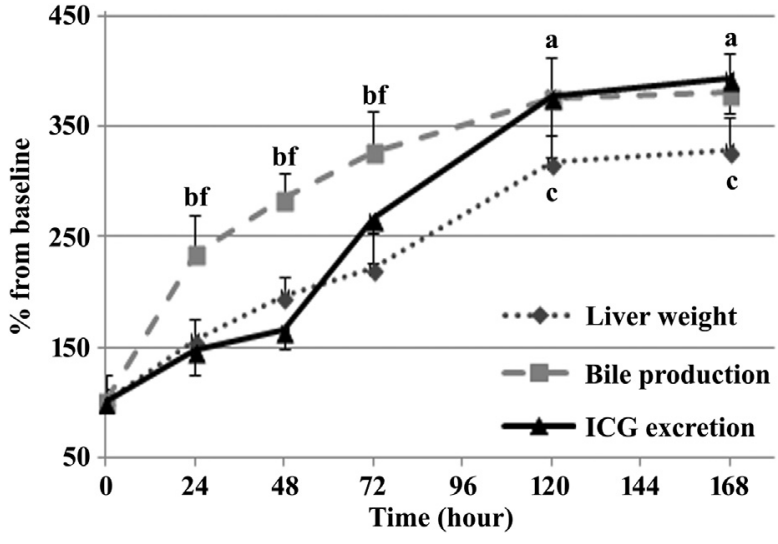

Fig. 6 - Comparison between morphologic and functional regeneration of PVNL lobes. The increment in bile production was more pronounced than the alteration in liver weight at each examined time point. In contrast, the increase in ICG excretion was smaller than the weight gain during the first $2 \mathrm{~d}$. From the third day, ICG excretion increased more dramatically than liver weight. Results are given as mean \pm standard deviation, $n=6$ per group. ${ }^{\mathrm{a} P}<0.05$ versus liver weight; ${ }^{b_{P}}<0.001$ versus liver weight; ${ }^{\mathrm{C}} \mathrm{P}<0.05$ versus ICG excretion; and ${ }^{\mathrm{f}} \mathrm{P}<0.001$ versus ICG excretion.

drainage to assess the alterations in lobar bile production and biliary ICG excretion.

Although total liver bile flow did not change significantly, portal vein ligation considerably affected bile production of the PVNL and PVL lobes. The volume of bile secreted by the PVL lobes gradually decreased after the operation. This progressive functional impairment, however, was fully counterbalanced by the excretory "overcompensation" of the PVNL lobes. The elevation of bile production in PVNL lobes exceeded the rapid increase in lobe weight. Consequently, the bile flow expressed per gram liver was significantly elevated after the operation, with the peak observable on the second day (1.5-fold increase). These findings are very similar to those described after partial hepatectomy, where bile flow in the remnant liver was also increased during the critical first $72 \mathrm{~h}$ of liver regeneration [23-26]. A quick adaptive response against the increased bile load can be assumed in the background. After portal vein ligation, all the portal blood arriving from the splanchnic organs is directed into the PVNL lobes as a result of which there is a significant increase in the amount of recirculating bile acids per gram liver [27]. Because bile acids are toxic at high concentrations, hepatocytes need to adapt to this challenge by increasing the biliary secretion of bile acids [24]. Under normal conditions, only periportal hepatocytes are involved in bile acid secretion; however, during liver regeneration, midzonal and pericentral hepatocytes also participate in bile acid transport [28]. The shift of bile acid processing toward the pericentral hepatocytes increases bile acid secretion per gram liver and may protect the mitotically active periportal cells against the toxic bile acid concentrations [23]. Because bile formation is based on the excretion of osmotically active bile acids, the increased bile acid secretion can explain the enhanced bile flow in the PVNL lobes.
Bile production, however, is not the most accurate indicator of liver function. Earlier studies had demonstrated that biliary ICG excretion shows strong correlation with intracellular ATP concentration [14]; it is therefore widely considered a more reliable indicator of liver function [11-13]. Consequently, in the present study, biliary ICG concentrations were assessed in addition to measuring bile production. The ICG excretory capacity of the total liver was temporarily impaired after the operation, with lowest value observed on the second day (nearly 50\% reduction) and a highly positive correlation $(P=0.87)$ apparent with the alterations detected in the PDR. This functional deterioration was presumably caused by the reduction in hepatobiliary transport of ICG, which appeared at varying degrees in both PVNL and PVL lobes.

The hepatobiliary transport of ICG is affected by several factors, such as serum bilirubin (competitive inhibition) and albumin (protein binding) levels, hepatic blood flow, influx across the sinusoidal membrane, intracellular transport, efflux across the canalicular membrane, as well as bile flow [29]. In the present study, the serum bilirubin and albumin levels did not change, whereas the microcirculatory blood flow and bile production of PVNL lobes showed considerable increase parallel with the rapid weight gain. Despite these alterations, the ICG excretion rate of PVNL lobes did not increase to a significant degree during the early phase of liver regeneration (days $1-2$ ). In the background of this finding, the impaired hepatocellular transport of the dye can be assumed. This assumption is supported by the analysis of the biliary ICG concentration curves, which demonstrated that the $\mathrm{C}_{\max }$ was decreased and the $\mathrm{T}_{\max }$ was prolonged after the operation. Considering that the cytoplasmic transport (microtubules) and canalicular excretion (multidrug resistance-associated protein 2) of ICG are ATP dependent, the previously mentioned alterations may indicate a decrease in the hepatic energy status of the PVNL lobes. This observation is in accordance with the results of Kameoka et al. [30] and Kucuktulu et al. [31], who demonstrated that the ATP concentration and metabolically available energy pool (energy charge) of PVNL lobes significantly decreased in the early phase of liver regeneration, caused by the enormous energy consumption of cell proliferation. In the present study, mitotic activity reached its peak on the second day, when $\mathrm{T}_{\max }$ and $\mathrm{C}_{\max }$ were the most impaired. After this critical point, the value of $C_{\max }$ and $T_{\max }$ gradually recovered, stabilizing slightly above the baseline level. Consequently, ICG excretion of the PVNL lobes showed a spectacular increase after the second day, which was much higher than the lobe weights. Analyzing hepatic ICG clearance function, Hashimoto et al. [32] demonstrated a similar "hyperfunction" of the remaining lobes after partial hepatectomy, which, however, was not considered as a real functional gain, but as a result of hyperdynamic circulation of the lobes. This hypothesis was not supported by our data. In the present study, the microcirculatory blood flow of PVNL did not differ from the preoperative value on the fifth and seventh days. Consequently, the observed "hyperfunction" at the end of liver regeneration (plateau phase) is most likely not flow dependent and may be explained by some other, presumably compensatory processes.

Portal vein ligation resulted in microcirculatory disorders in the PVL lobes. Accordingly, severe ischemia was notable 
in the centrolobular regions, leading to necrotic and apoptotic cell death and also to increased leukocytes infiltration. These circumstances (circulatory failure, cell loss, and inflammation) caused serious impairment in the lobar excretory function shortly after the operation. Nevertheless, from the second day, the microcirculation of the lobes showed gradual recovery, together with the return of normal hepatic histology. The functional capacity of the PVL lobes, however, remained permanently suppressed. On the seventh day, the liver lobules of the PVL lobes became considerably smaller and were characterized by contracted portal triads and narrowed sinusoids. Earlier, Tanaka et al. [21] and Yamasaki et al. [33] demonstrated that arterialization of the PVL lobes (absence of portal nutrients, hormones, and other mediators) led to the partial capillarization of liver sinusoids, characterized by a decreased number of fenestrae and porosity, as well as by partial development of the basement membrane. These alterations can reduce the diffusion of protein-bound substances such as ICG and are most likely responsible for the permanent functional impairment observed by us in the PVL lobes. Based on these observations, "hyperfunction" of the PVNL lobes can be a well-orchestrated compensatory response to the previously detailed functional atrophy of the PVL lobes.

\section{Conclusions}

In summary, portal vein ligation resulted in temporary impairment of total liver excretory function (indicated by ICG clearance test and biliary ICG excretion), caused by the functional deterioration of both PVL and PVNL lobes. This observation indicates that liver regeneration is initially promoted at the expense of the liver function. After the peak of cell division, however, an overcompensatory response was manifest in the PVNL lobes, during which the lobar liver function underwent more dramatic changes as compared with liver weight. Consequently, the functional capacity of the liver shifted toward the regenerating lobes in a greater extent than would have been expected according to the volumetric alterations, resulting in inhomogeneous functional distribution within the liver. These results indicate that in the early phase of liver regeneration, CT volumetry may overestimate the real alterations in liver function, whereas in the later phase of the regenerative process, these may be underestimated. Therefore, in addition to CT volumetry, monitoring of the hepatic function is strictly necessary to determine the regenerative status of the liver and the optimal waiting time for liver resection after PVO.

\section{Acknowledgment}

The authors thank Dr László Tretter and Dr Kraszimir Kolev (Department of Medical Biochemistry, Semmelweis University, Hungary) for their assistance in spectrophotometric measurements.

Authors' contributions: A.F., L.H., and A.S. conceived and designed the experiments. A.F., A.B., K.D., and S.P. performed the experiments. A.F., G.L., and Z.C. analyzed the data. G.L.,
K.D., and S.P. contributed reagents, materials, and analysis tools. A.F., Z.C., and A.S. wrote the article.

\section{Disclosure}

The authors report no proprietary or commercial interest in any product mentioned or concept discussed in this article.

\section{REFERENCES}

[1] EASL-EORTC clinical practice guidelines: management of hepatocellular carcinoma. J Hepatol 2012;56:908.

[2] Clavien PA, Petrowsky H, DeOliveira ML, Graf R. Strategies for safer liver surgery and partial liver transplantation. N Engl J Med 2007;356:1545.

[3] van Lienden KP, van den Esschert JW, de Graaf W, et al. Portal vein embolization before liver resection: a systematic review. Cardiovasc Interv Radiol 2013;36:25.

[4] Izuishi K, Wakabayashi H, Kokudo Y, Maeba T, Ryu M, Maeta H. Microsomal cytochrome P-450 monooxygenase system and its drug-metabolizing activity after partial portal vein ligation in the rat. World J Surg 1999;23:6.

[5] Takemura S, Minamiyama Y, Hirohashi K, Kubo S, Funae Y, Kinoshita H. Recovery of hepatic function determined by cytochrome P450-dependent drug metabolism lags after compensatory hepatic volume changes after portal vein ligation in rats. J Surg Res 2006;134:285.

[6] Nanashima A, Tobinaga S, Abo T, et al. Relationship of hepatic functional parameters with changes of functional liver volume using Technetium-99m galactosyl serum albumin scintigraphy in patients undergoing preoperative portal vein embolization: a follow-up report. J Surg Res 2010;164:e235.

[7] de Graaf W, van Lienden KP, van den Esschert JW, Bennink RJ, van Gulik TM. Increase in future remnant liver function after preoperative portal vein embolization. Br J Surg 2011;98:825.

[8] Yumoto Y, Yagi T, Sato S, et al. Preoperative estimation of remnant hepatic function using fusion images obtained by (99m)Tc-labelled galactosyl-human serum albumin liver scintigraphy and computed tomography. BrJ Surg 2010;97:934.

[9] Beppu T, Hayashi H, Okabe H, et al. Liver functional volumetry for portal vein embolization using a newly developed 99mTc-galactosyl human serum albumin scintigraphy SPECT-computed tomography fusion system. J Gastroenterol 2011;46:938.

[10] Szijarto A, Hargitai B, Fischer S, Darvas K, Kupcsulik P. Twostaged procedure of portal ligation and hepatectomy monitored by ICG clearance. J Invest Surg 2009;22:63.

[11] Seifalian AM, El-Desoky H, Delpy DT, Davidson BR. Effects of hepatic ischaemia/reperfusion injury in a rabbit model of indocyanine green clearance. Clin Sci (Lond) 2002;102:579.

[12] Sergi C, Gross W, Mory M, Schaefer M, Gebhard MM. Biliarytype cytokeratin pattern in a canine isolated perfused liver transplantation model. J Surg Res 2008;146:164.

[13] Kortgen A, Paxian M, Werth M, et al. Prospective assessment of hepatic function and mechanisms of dysfunction in the critically ill. Shock 2009;32:358.

[14] Chijiiwa K, Mizuta A, Ueda J, et al. Relation of biliary bile acid output to hepatic adenosine triphosphate level and biliary indocyanine green excretion in humans. World J Surg 2002; 26:457.

[15] Fulop A, Szijarto A, Harsanyi L, et al. Demonstration of metabolic and cellular effects of portal vein ligation using multi-modal PET/MRI measurements in healthy rat liver. PLoS One 2014;9:e90760. 
[16] Suzuki S, Toledo-Pereyra LH, Rodriguez FJ, Cejalvo D. Neutrophil infiltration as an important factor in liver ischemia and reperfusion injury. Modulating effects of FK506 and cyclosporine. Transplantation 1993;55:1265.

[17] Papp V, Dezso K, Laszlo V, Nagy P, Paku S. Architectural changes during regenerative and ontogenic liver growth in the rat. Liver Transpl 2009;15:177.

[18] Dezso K, Papp V, Bugyik E, et al. Structural analysis of oval-cellmediated liver regeneration in rats. Hepatology 2012;56:1457.

[19] Johnson DR, Habeebu SS, Klaassen CD. Increase in bile flow and biliary excretion of glutathione-derived sulfhydryls in rats by drug-metabolizing enzyme inducers is mediated by multidrug resistance protein 2. Toxicol Sci 2002;66:16.

[20] Schoen JM, Wang HH, Minuk GY, Lautt WW. Shear stressinduced nitric oxide release triggers the liver regeneration cascade. Nitric oxide 2001;5:453.

[21] Tanaka H, Ikeda K, Matsuyama M, et al. Morphological and functional changes after portal vein occlusion in rats. $J$ hepato-biliary-pancreatic Surg 1997;4:365.

[22] Mueller L, Broering DC, Meyer J, et al. The induction of the immediate-early-genes Egr-1, PAI-1 and PRL-1 during liver regeneration in surgical models is related to increased portal flow. J Hepatol 2002;37:606.

[23] Vos TA, Ros JE, Havinga R, et al. Regulation of hepatic transport systems involved in bile secretion during liver regeneration in rats. Hepatology 1999;29:1833.

[24] Csanaky IL, Aleksunes LM, Tanaka Y, Klaassen CD. Role of hepatic transporters in prevention of bile acid toxicity after partial hepatectomy in mice. Am J Physiol Gastrointest Liver Physiol 2009;297:G419.

[25] Hoshino M, Hirano A, Hayakawa T, et al. Comparative studies on bile flow and biliary lipid excretion after bile-acid loading in normal and partially hepatectomized rats. Biochem J 1995;305(Pt 2):367.

[26] Klaassen CD. Comparison of the effects of two-thirds hepatectomy and bile duct ligation on hepatic excretory function. J Pharmacol Exp Ther 1974;191:25.

[27] Hoekstra LT, Rietkerk M, van Lienden KP, van den Esschert JW, Schaap FG, van Gulik TM. Bile salts predict liver regeneration in rabbit model of portal vein embolization. J Surg Res 2012;178:773.

[28] Baumgartner U, Sellinger M, Ruf G, Jehle L, Ihling C, Farthmann EH. Change of zonal bile acid processing after partial hepatectomy in the rat. J Hepatol 1995;22: 474.

[29] El-Desoky A, Seifalian AM, Cope M, Delpy DT, Davidson BR. Experimental study of liver dysfunction evaluated by direct indocyanine green clearance using near infrared spectroscopy. Br J Surg 1999;86:1005.

[30] Kameoka N, Chijiiwa K, Kozaki N, Makino I, Naito T, Tanaka M. Hepatic adenine nucleotides and DNA synthesis during the regenerative and atrophic process of the liver lobes after selective portal vein ligation. Eur Surg Res 1996; 28:212.

[31] Kucuktulu U, Alhan E, Tekelioglu Y, Ozekin A. The effects of pentoxifylline on liver regeneration after portal vein ligation in rats. Liver Int 2007;27:274.

[32] Hashimoto M, Sanjo K. Functional capacity of the liver after two-thirds partial hepatectomy in the rat. Surgery 1997;121:690.

[33] Yamasaki M, Ikeda K, Nakatani K, et al. Phenotypical and morphological alterations to rat sinusoidal endothelial cells in arterialized livers after portal branch ligation. Arch histology Cytol 1999;62:401. 